\title{
La variabilidad espacio-temporal de la disponibilidad de agua
}

Adrián Pedrozo Acuña.

Fecha de publicación: 25 de julio de 2021

En cuencas y acuiferos donde no se mide de forma adecuada, se abre la puerta a errores y problemas en la estimación del agua disponible para su apropiación antropogénica.

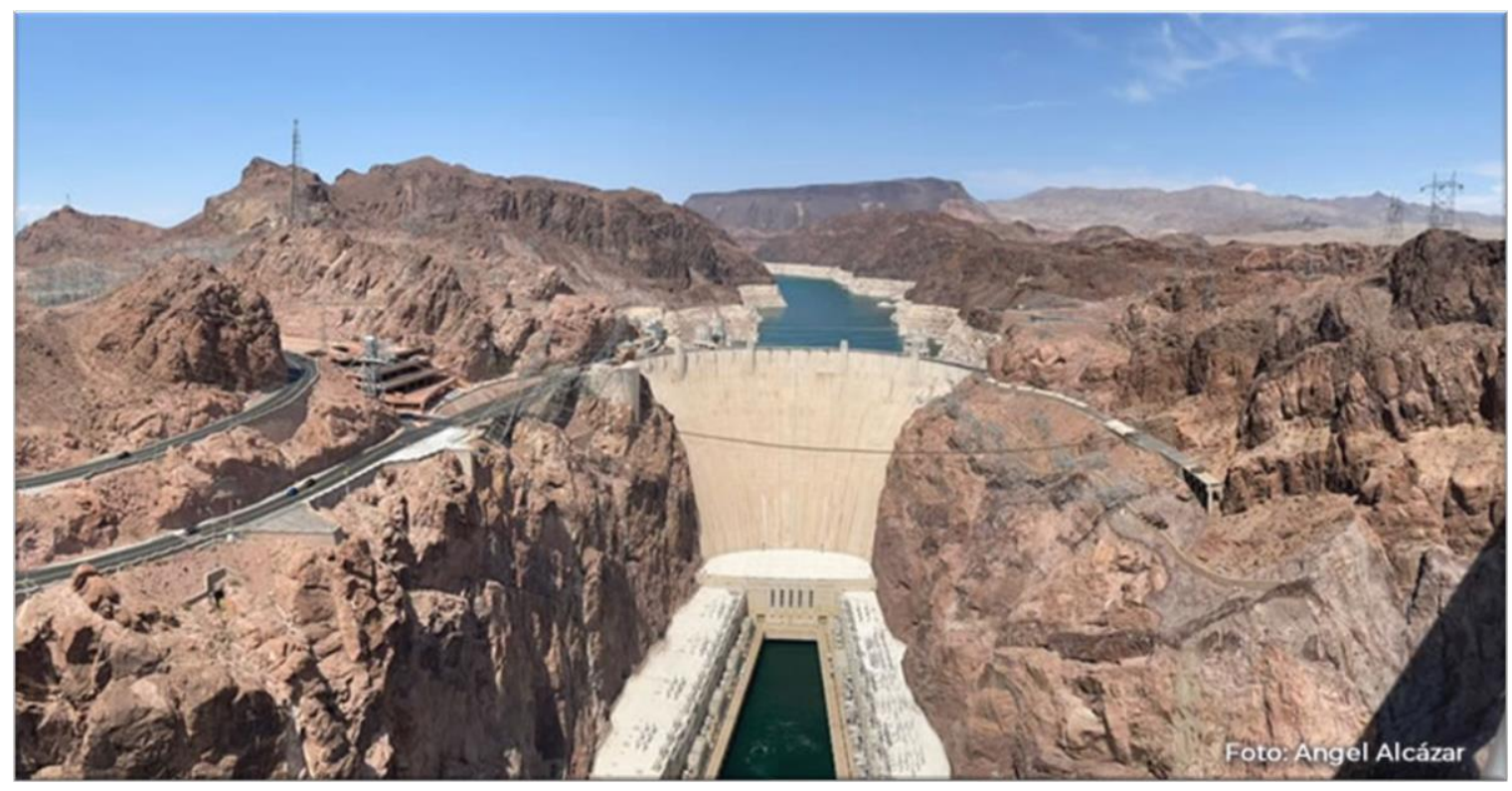

Durante el último siglo hemos visto que la interacción entre el crecimiento poblacional, el desarrollo económico continuo y la evolución de las dietas (hacia productos animales) ha resultado en una acelerada demanda de agua y, por tanto, en un incremento en la presión sobre esta.

Por ello, no es casualidad que varios lugares en el planeta estén experimentando una escasez hídrica, definida como una condición local y regional en la que el agua que se requiere para la operación segura de todos los sectores, incluyendo el medio ambiente, no está disponible, ya sea en cantidad o por un deterioro en su calidad (Vörösmarty et al., 2000). Hace algunos años, en su reporte de riesgos globales, el Foro Económico Mundial identificó la crisis en el abasto de agua como el riesgo de mayor impacto en el planeta (WEF, 2015).

Las diferentes asociaciones internacionales de agua e hidrología señalan la formulación de política pública para atender este tema como una de las prioridades más importantes de nuestros tiempos (Montanari et al., 2013). Se estima que cerca de 500 millones de personas se encuentran viviendo condiciones de escasez hídrica en todo el mundo (Mekonnen y Hoekstra, 2016). 
Dado que el futuro la población seguirá creciendo, junto con las necesidades para generar bienes y servicios, es necesario crear nuevos paradigmas de manejo y organización del agua. El punto central es que, actualmente, muchos lugares en el mundo exceden el consumo de agua al que se puede acceder de manera sustentable. La forma en la que usamos el agua excede de esta forma los límites naturales en diversos lugares del planeta (Hall et al., 2014). La lógica nos indica que una medida racional para limitar la sobreexplotación de nuestros cuerpos de agua sería justamente poner límites en términos de la cantidad de agua utilizada para producir un bien o servicio (p.ej. huella hídrica azul) por cuenca o acuífero (Hoekstra y Wieldmann, 2014; Hoekstra, 2017). Utilizando este marco de trabajo, Zhuo et al., (2019) analizaron el papel que desempeña la variabilidad temporal en la definición de una disponibilidad de agua, así como la gran incertidumbre asociada en la estimación del escurrimiento superficial y el requerimiento ambiental de un río (p. ej. gasto ecológico). Por otra parte, Grafton et al., (2014) revisaron, para el caso del río Murray-Darling, en Australia, el impacto de la variabilidad temporal en la definición de una disponibilidad hídrica para esta cuenca.

Pensar en la definición de límites a la huella hídrica para cada tipo de industria y actividad económica nos permite avanzar hacia la definición de umbrales ambientales y hacia el fomento de diferentes actividades económicas (p. ej. aquellas hídricamente intensivas) en lugares con mayor disponibilidad de agua. De esta manera, podríamos avanzar en la definición de límites de uso de agua que regionalmente y temporalmente, a lo largo del año, respeten los límites naturales impuestos por el planeta (Röckström et al., 2009). Esta idea es atractiva y tiene sentido, sin embargo, debemos reconocer que existen muchos obstáculos normativos y de juicio que previenen su implementación exitosa en todo el mundo.

Esto requiere la definición de una disponibilidad de agua dinámica en el tiempo (función de un clima que está cambiando), en contraste con la forma actual de considerar la disponibilidad estática y actualizarla cada cierto número de años. De esta manera se abre el acceso a la definición de meses en los que se tendrá una vigilancia más estricta en el uso (p. ej. estiaje o sequías), en contraste con las épocas del año en las que llueva más. Algunos investigadores han propuesto el cálculo de un límite en el volumen de agua a ser usado dentro de una cuenca en función de la variación mensual de la disponibilidad integrada (acuífero y cuenca). Por ejemplo, tomando en cuenta los promedios históricos de la disponibilidad integrada (cuenca y acuífero) a través de un promedio mensual, durante el periodo 1970-2005; el percentil $25^{\circ}$ del promedio mensual de la disponibilidad integrada, y la disponibilidad integrada mínima al mes, estimada en ese mismo periodo de tiempo (1970-2005) (Hogeboom et al., 2019).

Desde luego, estos estudios reconocen la gran cantidad de incertidumbre que está incorporada en sus resultados. Pero cabe mencionar que mantener el statu quo en la forma que definimos la disponibilidad de agua en nuestro país resultará en el mediano plazo en una fuente de conflictos sociales entre usuarios que requieren y requerirán acceso al agua (Steffen et al., 2015). A pesar de ello, es claro que la definición de límites variables en el tiempo y en regiones es más significativa desde un punto de vista físico, que considerar condiciones estáticas $\mathrm{y}$, por tanto, tienen mayor probabilidad de éxito en su utilización para la implementación de nuevas políticas públicas del agua (Heck et al., 2018).

Dentro de los retos que ya se anticipan para la implementación adecuada de un marco de trabajo en este sentido, está la cuantificación precisa de las extracciones de agua subterránea en nuestros acuíferos, respetando una estimación más precisa de la recarga real. Por otro lado, es probable que sea necesario considerar también la variación espacial de la disponibilidad del agua en acuíferos de gran extensión territorial, además de considerar su alineación con la correspondiente jurisdicción administrativa. Otros temas que será necesario tener en mente son los asociados a la distribución 
PERSPECTIVAS IMTA

$N^{\circ} .28,2021$

Autor: Adrián Pedrozo Acuña

DOI: doi.org/10.24850/b-imta-perspectivas-2021-28

equitativa del agua entre usuarios, comunidades y medio ambiente. Esto requiere, además, el mejoramiento de los sistemas de medición de nuestros cuerpos de agua y las metodologías utilizadas para la estimación de los balances hídricos. En cuencas y acuíferos donde no se mide de forma adecuada, se abre la puerta a errores y problemas en la estimación del agua disponible para su apropiación antropogénica, que, con el tiempo, tienen el potencial de generar conflictos sociales. Esto es aún más cierto en aquellos lugares del territorio donde ya se tiene identificada la sobreexplotación del agua, o en cuencas o acuíferos en los que hay una alta variabilidad climática.

Para todo el mundo, el reto del siglo XXI es mover nuestra política hídrica hacia la sustentabilidad y el respeto a la vida. Limitar nuestro uso y apropiación del agua, en función de lo que el territorio y la naturaleza nos definen, no tiene por qué limitar nuestro desarrollo económico como nación. Pero para ello, debemos mudar la forma en la que pensamos y atendemos los problemas relativos al agua. En el Instituto Mexicano de Tecnología del Agua lo estamos haciendo, por el bien de nuestro país.

\section{Referencias}

Grafton, R. Q., Pittock, J., Williams, J., Jiang, Q., Possingham, H., \& Quiggin, J. (2014). Water planning and hydro-climatic change in the Murray-Darling Basin, Australia. Ambio, 43(8), $1082-1092$. https://doi.org/10.1007/s13280-014-0495-x

Hall, J. W., Grey, D., Garrick, D., Fung, F., Brown, C., Dadson, S. J., \& Sadoff, C. W. (2014). Coping with the curse of freshwater variability. Science, 346(6208), 429-430. https://doi.org/10.1126/science.1257890

Heck, V., Hoff, H., Wirsenius, S., Meyer, C., \& Kreft, H. (2018). Land use options for staying within the planetary boundaries-Synergies and trade-offs between global and local sustainability goals. Global Environmental Change, 49, 73-84.

Hoekstra, A. Y. (2017). Water footprint assessment (WFA): Evolvement of a new research field. Water Resources Management, 31(10), 3061- 3081.

Hoekstra, A. Y., \& Wiedmann, T. O. (2014). Humanity's unsustainable environmental footprint. Science, 344(6188), 1114- 1117. https://doi.org/10.1126/science.1248365

Hogeboom, R. J., de Bruin, D., Schyns, J. F., Krol, M. S., \& Hoekstra, A. Y. (2019). Capping human water footprints in the world's river basins, Mendeley Data, v1. https://doi.org/10.17632/n97vjpxhj2.1

Mekonnen, M. M., and A. Y. Hoekstra (2016), Four billion people facing severe water scarcity, Sci. Adv., 2, e1500323. https://doi.org/10.1126/sciadv.1500323.

Montanari, A., et al. (2013), "Panta Rhei, Everything Flows": Change in hydrology and society; the IAHS scientific decade 2013-2022, Hydrol. Sci. J., $\quad 58(6), \quad 1256-\quad 1275$. https://doi.org/10.1080/02626667.2013.809088.

Rockström, J., Steffen, W., Noone, K., Persson, Å., Chapin, F. S., Lambin, E. F., Lenton, T. M., Scheffer, M., Folke, C., Schellnhuber, H. J., Nykvist, B., De Wit, C. A., Hughes, T., Van Der Leeuw, S., Rodhe, H., Sörlin, S., Snyder, P. K., Costanza, R., Svedin, U., Falkenmark, M., Karlberg, L., Corell, R. W., Fabry, V. J., Hansen, J., Walker, B., Liverman, D., Richardson, K., Crutzen, P., \& Foley, J. A. (2009). A safe operating space for humanity. Nature, 461(7263), 472- 475. https://doi.org/10.1038/461472a

Steffen, W., Richardson, K., Rockstrom, J., Cornell, S. E., Fetzer, I., Bennett, E. M., Biggs, R., Carpenter, S. R., de Vries, W., de Wit, C. A., Folke, C., Gerten, D., Heinke, J., Mace, G. M., Persson, L. M., Ramanathan, V., Reyers, B., \& Sorlin, S. (2015). Sustainability. Planetary boundaries: Guiding human development on a changing planet. Science, 347(6223), 1259855.

Vörösmarty, C. J., McIntyre, P. B., Gessner, M. O., Dudgeon, D., Prusevich, A., Green, P., Glidden, S., Bunn, S. E., Sullivan, C. A., Liermann, C. R., \& Davies, P. M. (2010). Global threats to human water security and river biodiversity. Nature, 467(7315), 555- 561. https://doi.org/10.1038/nature09440 
PERSPECTIVAS IMTA (0)

$\mathrm{N}^{\circ} .28,2021$

Autor: Adrián Pedrozo Acuña

DOI: doi.org/10.24850/b-imta-perspectivas-2021-28

World Economic Forum (2015), Global Risks 2015, 10th ed., pp. , World Economic Forum, Geneva, Switzerland.

Zhuo, L., Hoekstra, A. Y., Wu, P., \& Zhao, X. (2019). Monthly blue water footprint caps in a river basin to achieve sustainable water consumption: The role of reservoirs. Science of the Total Environment, $650(\mathrm{Pt}$ 1), 891- 899. 